Number of pages: 14

Number of references: 24

Number of Figures: 11

\title{
Postural stability when walking: effect of the frequency and magnitude of lateral oscillatory motion
}

\author{
Mujde Sari and Michael J. Griffin \\ Human Factors Research Unit \\ Institute of Sound and Vibration Research \\ University of Southampton \\ Southampton SO17 1BJ \\ United Kingdom
}

Corresponding author: Michael J. Griffin (M.J.Griffin@soton.ac.uk) 


\begin{abstract}
While walking on an instrumented treadmill, 20 subjects were perturbed by lateral sinusoidal oscillations representative of those encountered in transport: frequencies in the range 0.5 to 2 $\mathrm{Hz}$ and accelerations in the range 0.1 to $2.0 \mathrm{~ms}^{-2}$ r.m.s., corresponding to velocities in the range 0.032 to $0.16 \mathrm{~ms}^{-1}$ r.m.s. Postural stability was assessed from the self-reported probability of losing balance (i.e., perceived risk of falling) and the movements of the centre of pressure beneath the feet. With the same acceleration at all frequencies, the velocities and displacements of the oscillatory perturbations were greater with the lower frequency oscillations, and these caused greater postural instability. With the same velocity at all frequencies, postural instability was almost independent of the frequency of oscillation. Movements of the centre of pressure show that subjects attempted to compensate for the perturbations by increasing their step width and increasing their step rate.
\end{abstract}

Keywords: postural stability; walking; lateral oscillatory motion 


\section{Introduction}

Standing and walking require continuous postural control to counteract the destabilizing effects of gravity and self-induced movements of the body. Maintaining balance is more challenging when there are external disturbances from motion of the floor, such as when standing or walking in a moving train, bus, aircraft or ship.

Sudden accelerations or decelerations of a treadmill (Berger et al., 1984) or a moveable platform embedded in a walkway (e.g., Nashner, 1980; Oddsson et al., 2004; Bhatt et al., 2005) have been used to investigate responses to slips, trips, and missteps encountered during walking. Longer duration low frequency oscillations $(0.2$ to $0.5 \mathrm{~Hz})$ of a treadmill on a six-axis motion platform (e.g., Brady et al., 2009; McAndrew et al., 2010) have been used to investigate postural responses to perturbations when walking but have not been representative of motions encountered in transport.

With instantaneous increases in horizontal acceleration, stationary standing people have been reported to tolerate accelerations up to $0.76 \mathrm{~ms}^{-2}$ in the backward direction, $0.48 \mathrm{~ms}^{-2}$ in the forward direction, and $0.33 \mathrm{~ms}^{-2}$ in a sideways direction (Jongkees and Groen, 1942 - as cited by Graaf and Weperen, 1997). Similar thresholds were obtained by Graaf and Weperen (1997), who found that standing subjects were most sensitive to lateral acceleration when standing with their feet almost together. Postural stability when standing still and exposed to narrow-band random fore-and-aft and lateral oscillation has been studied at frequencies between 0.125 and $2.0 \mathrm{~Hz}$ with velocities from 0.04 to $0.16 \mathrm{~ms}^{-1}$ r.m.s. (Nawayseh and Griffin, 2006). The displacement of the centre of pressure (COP) and subject estimates of the probability of losing balance increased with increasing magnitude of oscillation and, with the same velocity at all frequencies, stability problems were greatest around $0.5 \mathrm{~Hz}$. Tolerances of walking subjects to sideward oscillations in transport have not been previously reported.

Understanding of the physiological and biomechanical aspects of balance has been used to develop active models of postural stability when standing (e.g. Mergner et al., 2006; Peterka 2003). These models represent the neural, sensory, and biomechanical subsystems involved in human postural control but do not allow the prediction of the probability of falling. People may be expected to be more stable when standing and supported on two legs than when walking and supported on only one leg for $80 \%$ of the gait cycle (Woollacott and Tang, 1997), especially when threatened by external perturbations.

The main strategy used to maintain balance during locomotion is the stepping strategy (Nashner, 1980; Horak and Nashner,1986; Hof et al., 2007). Additional strategies (e.g. active hip torque and active ankle subtalar torque) are used for fine tuning (Hof et al., 2007; MacKinnon and Winter, 1993) when the foot position is established. Adjusting the step width by varying the foot placement maintains postural stability in the frontal (i.e., coronal) plane by regulating the trajectory of the centre of mass (COM) (Townsend, 1985) and has a greater influence on postural control during unperturbed walking than either step length or step time 
(Owings and Grabiner, 2004). It has been suggested that step width is adjusted to compensate for lateral acceleration induced by external perturbation (Oddsson et al., 2004).

The overall aim of the study reported in this paper was to determine how postural stability when walking is influenced by the magnitude and the frequency of lateral oscillation of the floor. It was hypothesised that, at each frequency of oscillation, the self-reported probability of losing balance and the movement of the centre of pressure in the lateral direction would increase with increasing magnitude of oscillation. It was expected that the movement of the centre of pressure would vary with the frequency of oscillation in a manner that would explain the frequency-dependence of the loss of balance.

\section{Method}

\subsection{Subjects}

Twenty healthy male subjects median age 27 years (range 25 to 41 ), stature $177 \mathrm{~cm}$ (range 165 to 192), weight $72.3 \mathrm{~kg}$ (48.5 kg to 88.45 ) participated in the study. Subjects completed a questionnaire to exclude those with relevant disorders or using drugs that might affect postural stability. Informed consent was obtained prior to participation in the experiment that was approved by the Human Experimentation Safety and Ethics Committee of the Institute of Sound and Vibration Research.

\subsection{Apparatus}

A treadmill (Kistler Gaitway ${ }^{\circledR}$ ) incorporating eight force sensors was used to provide the walking task and measure the vertical ground reaction forces during walking. Subjects were secured by a safety harness connected via two loose straps to a frame around the treadmill (Fig. 1). The harness allowed subjects to move freely in the plane of progression but prevented their knees or hips contacting the floor if they fell. A safety net was positioned behind the subjects as a precaution in case they slid backwards while walking on the treadmill.

\section{FIGURE 1 ABOUT HERE}

Lateral oscillatory motion was generated by a six-axis motion simulator in the Human Factors Research Unit at the Institute of Sound and Vibration Research. The simulator is able to provide translational displacements of $\pm 0.5 \mathrm{~m}$ in the lateral direction at accelerations up to about $\pm 10 \mathrm{~ms}^{-2}$.

Acceleration in the lateral direction was recorded by accelerometers on the simulator platform (FGP model FA101-A2-5G). Data acquisition via the treadmill software was triggered at the moment the $4 \frac{1}{2}$-cycle acceleration commenced. The acceleration and force data collected by the Gaitway ${ }^{\circledR}$ data acquisition system were sampled at 100 samples per second and stored in a personal computer.

\subsection{Experimental Procedure}


While walking on the treadmill, subjects were perturbed by simple transient lateral acceleration stimuli applied at an unpredictable time. The stimuli were 4.5 cycles of sinusoidal motion modulated by a half sine envelope. For these waveforms, the peak acceleration and the peak velocity are, respectively, double the r.m.s. acceleration and r.m.s. velocity. The motions start and end with zero displacement, velocity and acceleration and were chosen as being broadly representative of lateral motions experienced in trains (Fig. 2a).

\section{FIGURE 2 ABOUT HERE}

At each of seven frequencies $(0.5,0.63,0.8,1.0,1.25,1.6,2.0 \mathrm{~Hz})$, the motions were presented at eight velocities $\left(0.032,0.04,0.05,0.062,0.08,0.1,0.125,0.16 \mathrm{~ms}^{-1}\right.$ r.m.s. $)$. This resulted in accelerations in the range 0.1 to $2.0 \mathrm{~ms}^{-2}$ r.m.s. (Fig. 2b). The frequencies and magnitudes were chosen after preliminary experimentation and so that the effects of stimuli with the same magnitude of acceleration or stimuli with the same magnitude of velocity could be compared across the frequency range. The 56 motions were presented in a random order.

The speed of the treadmill was selected so that subjects walked at $0.7 \mathrm{~ms}^{-1}$ throughout the experiment. This was the preferred comfortable walking speed of subjects who participated in preliminary experiments.

The eight channels of force data were acquired throughout each of the $4 \frac{1}{2}$-cycle perturbations. After experiencing each motion, subjects were asked to judge their postural stability by answering the following question:

"What is the probability that you would lose balance if the same exposure were repeated?"

Subjects were instructed to fix their vision on the board in front of them (which moved with the lateral motion of the simulator) and to grasp the handrails of the treadmill only if it was really necessary. Losing balance was defined as attempting to take protective action not to fall such as taking a protective step, or grasping an object to regain equilibrium.

\subsection{Analysis}

The raw force time-histories (from 8 force sensors) were processed to determine the centre of pressure (COP) during each motion. The arrangement of the force sensors in the treadmill is shown in Fig. 3. The COP in the lateral direction was obtained by moment equilibrium of the vertical ground reaction forces gathered via eight force sensors with respect to longitudinal axis of the treadmill (x-axis, Fig.3):

FIGURE 3 ABOUT HERE

$$
\operatorname{COP}_{\mathrm{y}}=\frac{\mathrm{a}\left(F_{1}+F_{4}+F_{5}+F_{8}\right)-\mathrm{a}\left(F_{2}+F_{3}+F_{6}+F_{7}\right)}{F_{1}+F_{2}+F_{3}+F_{4}+F_{5}+F_{6}+F_{7}+F_{8}}
$$

Where the distance, $a$, and the forces, $F$, are defined in Fig. 3. 
The COP velocity in the lateral direction was obtained by differentiating the lateral COP position after filtering the centre of pressure position using a low-pass Bessel filter at $8 \mathrm{~Hz}$.

An example of the COP position and COP velocity of a subject exposed to $0.8-\mathrm{Hz}$ lateral oscillation at $0.5 \mathrm{~ms}^{-2}$ r.m.s. is shown in Fig. 4 . The mean value has been subtracted from the COP position, which shows the location of the ground reaction forces in the lateral direction $(y$-axis) and is indicative of lateral foot placement. The COP velocity indicates the rate of change of COP position (Fig. 4b).

\section{FIGURE 4 ABOUT HERE}

Non-parametric statistical methods were used for the data analysis using SPSS (version 17). The Friedman analysis of variance was used to test for differences between multiple conditions and the Wilcoxon matched-pairs signed ranks was used to test for differences between pairs of conditions. Associations between variables were investigated using Spearman's rank correlation.

\section{Results}

\subsection{Subjective Data}

At each frequency, the median reported probability of losing balance increased as the acceleration or the velocity of the lateral motion increased $(p<0.01$ at all seven frequencies; Spearman; Fig. 5). When the motion magnitude was expressed in terms of velocity, the perceived risk of fall was broadly similar at all frequencies and at all magnitudes (Fig. 5b). The inter-quartile range varied from $8 \%\left(\right.$ at $0.032 \mathrm{~ms}^{-1}$ ) to $40 \%\left(\right.$ at $0.1 \mathrm{~ms}^{-1}$ ).

\section{FIGURE 5 ABOUT HERE}

At each acceleration magnitude, the perceived risk of fall decreased as the frequency of oscillation increased $\left(p<0.01\right.$ at $0.125,0.16,0.315,0.4,0.5,0.63,0.8,1.0,1.25$, and $1.6 \mathrm{~ms}^{-2}$ r.m.s.; $p<0.05$ at 0.2 and $0.25 \mathrm{~ms}^{-2}$ r.m.s.; Spearman; Fig. $\left.6 a\right)$.

At each velocity magnitude, the median reported probability of losing balance was similar at all frequencies, with a significant effect of frequency at only two magnitudes $(p<0.05$ at 0.08 $\mathrm{ms}^{-1}$ r.m.s.; $p<0.01$ at $0.13 \mathrm{~ms}^{-1}$ r.m.s.; Friedman; Fig. $6 \mathrm{~b}$ ). Within the frequency range 0.63 to $1.6 \mathrm{~Hz}$ there was no significant effect of frequency on the probability of losing balance at any magnitude of velocity ( $p>0.06$, Friedman).

\section{FIGURE 6 ABOUT HERE}

The number of subjects, $N$, estimating their probability of losing balance to be $50 \%$ or greater was counted and related to the magnitude of the oscillation. The accelerations required at each frequency for $25 \%, 50 \%$ and $100 \%$ of the 20 subjects to report their probability of losing balance to be $50 \%$ or greater are shown in Fig. 7 .

\section{FIGURE 7 ABOUT HERE}




\subsection{Objective Data}

The peak-to-peak lateral COP position was used as a measure of the lateral foot placement and the lateral r.m.s. COP velocity was used as a measure of the timing of stepping action.

With each frequency of lateral acceleration, the peak-to-peak lateral COP position increased as the magnitude of the motion increased at all frequencies $(p<0.01$, Spearman; Fig. 8) except at $0.63 \mathrm{~Hz}(p=0.091$, Spearman). Changes in the peak-to-peak COP position were positively correlated with changes in the reported probability of losing balance at each frequency of oscillation ( $p<0.01$, Spearman), except at $0.63 \mathrm{~Hz}(p=0.1$, Spearman).

\section{FIGURE 8 ABOUT HERE}

At each acceleration magnitude, the peak-to-peak lateral COP position decreased as the frequency of oscillation increased ( $p<0.01$, Spearman; Fig. 9), except at $0.2 \mathrm{~ms}^{-2}$ r.m.s. $(p=0.1$, Spearman). The peak-to-peak lateral COP position was correlated with the reported probability of losing balance at each acceleration magnitude ( $p<0.025$, Spearman).

\section{FIGURE 9 ABOUT HERE}

When the motion was applied at the same velocity, the peak-to-peak lateral COP position decreased with increasing frequency ( $p<0.05$, Spearman), although the reported probability of losing balance was similar (Fig. 6b). However, the r.m.s. COP velocity showed a trend similar to the subjective ratings of postural stability: the r.m.s. COP velocity was not correlated with the frequency of oscillation at any magnitudes of lateral velocity ( $p>0.5$, Spearman; Fig. 10), except for a positive correlation with frequency at $0.08 \mathrm{~ms}^{-1}$ r.m.s. ( $p=0.014$, Spearman).

\section{FIGURE 10 ABOUT HERE}

The lateral r.m.s. COP velocity increased with increasing lateral velocity at all frequencies $(p<0.05$, Spearman; Fig.11), except at $0.63 \mathrm{~Hz}$. The increase in lateral r.m.s. COP velocity with increasing velocity magnitude was broadly similar at all frequencies and similar to the trend in the reported probability of losing balance with increasing velocity of oscillation (Fig. $5 b)$.

\section{FIGURE 11 ABOUT HERE}

During unperturbed walking, the median lateral peak-to-peak COP position and r.m.s. COP velocity were $18.42 \mathrm{~cm}$ and $35.79 \mathrm{cms}^{-1}$, respectively. The peak-to-peak lateral COP position during unperturbed walking was less than when walking and perturbed by lateral oscillation at any frequency and magnitude of oscillation ( $p<0.05$, Wilcoxon), except two conditions with low magnitude lateral oscillation at high frequencies $\left(0.032 \mathrm{~ms}^{-1}\right.$ r.m.s. with $1.6-\mathrm{Hz}$ oscillation, and $0.04 \mathrm{~ms}^{-1}$ r.m.s. with $2-\mathrm{Hz}$ oscillation; $p>0.05$, Wilcoxon). The lateral r.m.s. COP velocity during unperturbed walking was less than when walking and perturbed by lateral oscillation at any frequency and magnitude of oscillation ( $p<0.05$, Wilcoxon), except two conditions with low magnitude lateral oscillation $\left(0.032 \mathrm{~ms}^{-1}\right.$ r.m.s. with $1.6 \mathrm{~Hz}$ oscillation, and $0.05 \mathrm{~ms}^{-1}$ r.m.s. with $0.63 \mathrm{~Hz}$ oscillation; $p>0.05$, Wilcoxon). 


\section{Discussion}

With all frequencies of lateral oscillation, as the magnitude of the perturbation increased the perceived risk of fall increased (Fig. 5). The peak-to-peak lateral COP position and the r.m.s. lateral COP velocity also increased with increased magnitude of oscillation (Fig. 8 and Fig. 11). The increase in peak-to-peak COP position indicates an increase in step width, which is adjusted to compensate for lateral acceleration induced by external perturbations (e.g., Oddsson et al., 2004; Brady et al., 2009). The increase in lateral r.m.s. COP velocity with increasing magnitude of oscillation indicates that subjects used faster stepping to counteract the destabilising effects of the lateral oscillation. Random oscillation of a floor has also been reported to cause wider and faster stepping (McAndrew et al., 2010).

With the same acceleration at all frequencies, the stability of stationary standing people perturbed by lateral sinusoidal oscillation is less affected by higher frequencies (e.g. 0.5 to 2 $\mathrm{Hz}$ ) than by lower frequencies (e.g. 0.125 to $0.5 \mathrm{~Hz}$ ) (Nawayseh and Griffin, 2006). Over the same frequency range, the current study also found less postural instability (reduced probability of losing balance and reduced peak COP displacement) as the frequency of oscillation increased with constant magnitude of acceleration (Fig. 6a and Fig. 9). Stationary standing people perturbed by lateral oscillations with the same velocity showed greatest displacements of the centre of pressure and greatest subjective estimates of the probability of losing balance around $0.5 \mathrm{~Hz}$ (Nawayseh and Griffin, 2006). In the present study, irrespective of the frequency of oscillation, the probability of losing balance in walking subjects was broadly similar when the lateral oscillation was applied at the same r.m.s. velocity (Fig. 6b). The peak COP displacement (i.e., peak-to-peak COP position) decreased with increasing frequency of oscillation but the r.m.s. COP velocity was broadly independent of the frequency of oscillation at the same r.m.s. velocity (Fig. 10). The lateral r.m.s. COP velocity may indicate effort to respond to the velocity of external perturbations by adjusting the timing of foot placement.

With the same acceleration at all frequencies, there are greater velocities and greater displacements with the lower frequency oscillations. The results of the present study show these motions cause greater difficulty in adjusting the centre of pressure, presumably because the motions are larger and faster. In response to a movement of the centre of mass, the centre of pressure is adjusted using postural control strategies (Murray et al. 1967; Prieto et al. 1993). Walking people are sensitive to changes in lateral velocity and take corrective stepping actions (Hof, 2008; Hof et al. 2010). The findings of the present study are consistent with walking people responding to changes in lateral velocity by adjusting their lateral COP velocity.

Stability thresholds have not previously been reported for walking subjects. Arbitrarily, the findings of this study have been used to calculate the magnitudes required at each frequency of lateral oscillation for $50 \%$ of subjects to report at least $50 \%$ probability of losing balance 
(Fig. 7). Subjects standing with their eyes closed and their feet together have been reported to tolerate 'step' changes in lateral acceleration (sudden constant acceleration followed by a constant deceleration) up to $\pm 0.33 \mathrm{~ms}^{-2}$ r.m.s. (Jongkees and Groen, 1942). In the current study, an acceleration of about $0.3 \mathrm{~ms}^{-2}$ r.m.s. at about $0.5 \mathrm{~Hz}$ resulted in about $50 \%$ of subjects reporting at least $50 \%$ probability of losing balance, but a much greater acceleration was required to produce the same effect with the higher frequencies of oscillation (Fig. 5a). A stability threshold of $\pm 0.45 \mathrm{~ms}^{-2}$ has been reported for subjects standing with their hands free, heels together, and toes 3 to $4 \mathrm{~cm}$ apart while exposed to sudden acceleration or deceleration without holding handrails, or taking a protective step, or stabilizing the body by large body sways or arm movements (Graaf and Weperen, 1997). In addition to the use of standing as opposed to walking subjects, and some other important details, these previous studies (Jongkees and Groen, 1942; Graaf and Weperen, 1997) differ in respect of the waveform of the motion stimulus. The present results show that the effects of lateral acceleration on postural stability are highly frequency-dependent and cannot be predicted solely from the peak acceleration although, for the waveforms investigated, stability is well predicted by both the peak velocity and the r.m.s. velocity.

Dynamic balance during normal locomotion is mainly achieved by adjusting the timing and placement of successive steps (Nashner, 1980). To compensate for medio-lateral acceleration induced by perturbations, it has been suggested that the central nervous system adjusts the step width to alter the moment arm (Oddsson et al., 2004). Although the main strategy for maintaining balance is the 'stepping strategy', large errors in foot placement are corrected by hip moments (Hof et al., 2007; McKinnon and Winter 1993) and fine tuning is achieved by active ankle moments. The overall effects of the magnitude and frequency of oscillation on lateral COP movement in the present study implies that most of the subjects primarily used stepping strategies to counteract the destabilizing effects of lateral oscillation.

There are differences between walking on a treadmill and walking along a floor. Subjects could not stop walking when their stability was threatened and so, unlike in many forms of transport, remaining stationary for a period of time was not an acceptable response. Otherwise, the biomechanics of walking on a treadmill and walking on a floor may be similar (Wagenaar and Beek, 2000), although the use of a speed preferred by the subject or the same speed controlled for all subjects has been reported to affect postural responses to perturbations during gait (Duysens and Bloem, 2009). The controlled speed of $0.7 \mathrm{~ms}^{-1}$ used in the present study was judged to be a comfortable walking speed by subjects in preliminary experiments but stability may differ with faster or slower speeds.

The perceived risk of falling reported in this study may differ from the risk of passengers falling in transport. The subjects were prevented from falling, and so their reported probability of falling was influenced by the extent to which they found it necessary to take protective action, rather than by experiencing a fall. The lateral COP displacement and r.m.s. COP velocity may primarily reflect subject effort to continue walking by compensating with a wider 
or quicker step when motion threatened their stability. Although the subjective and objective measures of postural stability used in this study reflect threats to postural stability, with the same motions in transport the risk of falling may differ for a variety of reasons (e.g., because the attention of passengers may not be focused on preventing falls and the risk of fall will be greater without a safety harness and handrail). The stability thresholds provided for lateral oscillations that are typical of motions in some trains indicate the dependence of postural stability on the frequency of oscillation and may also provide an indication of the likely effects of motion on the stability of people walking in transport.

The present study was conducted with fit young male subjects who volunteered to participate in the study. Large differences in postural stability when walking and exposed to perturbations are expected to be associated with differences in age, gender, balance disorders, fitness, clothing, and carrying. The population participating in the study may be assumed to be among those least affected by motion perturbations: greater problems may be expected with some members of the general public, some of whom may be deterred from travelling by the risk of falling when moving around during travel.

\section{Conclusion}

The postural instability caused by low frequency lateral oscillations over the frequency range 0.5 to $2 \mathrm{~Hz}$, typical of those experienced in some forms of transport, cannot be predicted solely from the lateral acceleration. For transient sinusoidal oscillations of the type studied, postural instability may be predicted from the velocity of the motion. Movements of the centre of pressure show that subjects attempt to compensate for perturbations by increasing their step width and increasing their step rate.

\section{Acknowledgements}

This study was conducted with the support of Sociéte Nationale des Chemins de fer Français (SNCF), Paris, France. The assistance of Cedric Gallais is gratefully acknowledged.

\section{References}

Berger, W., Dietz, V., Quintern, J., 1984. Corrective reactions to stumbling in man: Neuronal coordination of bilateral leg muscle activity during gait. The Journal of Physiology 357, 109125.

Nashner, L.M., 1980. Balance adjustments of humans perturbed while walking. Journal of Neurophysiology 44, () 650-664.

Oddsson, L.I.E., Wall III, C., McPartland, M.D., Krebs, D.E. , Tucker, C.A., 2004. Recovery from perturbations during paced walking. Gait and Posture 19, 24-34.

Bhatt, T., Wening, J.D., Pai, Y.-C., 2005. Influence of gait speed on stability: recovery from anterior slips and compensatory slipping. Gait and Posture 21,146-156. 
Brady, R.A., Peters, B.T. and Bloomberg, J.J., 2009. Strategies of healthy adults walking on a laterally oscillating treadmill. Gait and Posture 29, 645-649.

Duysens, J., Bloem, B., 2009. Preferring preferred speed, Proceedings of the Nineteenth Conference of the International Society for Posture and Gait Research, Bologna, Italy, June 2009, 40.

Nashner, L.M., 1986. Organization of human postural movements during standing and walking. In S. Grillner, P. Stein, D.G. Stuart, H. Forssberg and R.M. Herman (Eds.), Neurobiology of Vertebrate Locomotion, Wenner-Gren International Symposium-Series, London, MacMillan, 637-648.

McAndrew, P.M., Dingwell, J.B. and Wilken, J.M., 2010. Walking variability during continuous pseudo-random oscillations of the support surface and visual field. Journal of Biomechanics 43, 1470-1475.

Nawayseh, N., Griffin, M.J., 2006. Effect of frequency, magnitude and direction of translational and rotational oscillation on the postural stability of standing people. Journal of Sound and Vibration 298, 725-754.

Jongkees, L.B.W., Groen, J.J., 1942. De standvastigheid van de mens [The stability of the human body]. Nederlands Tiidschrift voor Geneeskunde 86,1401-1407.

Graaf, B. De, Van Weperen, W., 1997. The retention of balance: An explanatory study into the limits of acceleration without losing equilibrium. Human Factors and Ergonomics Society, 39(1),111-118.

Mergner, T., Schweigart, G., Maurer, C., Blumle, A., 2006. Human postural responses to motion of real and virtual visual environments under different support base conditions. Experimental Brain Research 167, 535-556.

Peterka, R.J., 2003. Simplifying the complexities of maintaining balance. IEEE Engineering in Medicine and Biology Magazine 22,63-68.

Woollacott, M.H., Tang, P., 1997. Balance control during walking in the older adult: Research and its implications. Physical Therapy 77, 646-660.

Horak, F.B., Nashner, L.M., 1986. Central programming of postural movements: adaptation to altered support-surface configurations. Journal of Neurophysiology 55,1369-1381.

Hof, At.L., van Bockel, R.M., Schoppen, TPostema, ., K., 2007. Control of lateral balance in walking: Experimental findings in normal subjects and above-knee amputees. Gait and Posture 25,250-258.

Hof, A. L., 2008. The 'extrapolated center of mass' concept suggests a simple control of balance in walking. Human Movement Science 27, 112-125.

Hof, A. L., Vermerris, S.M., Gjaltema, W.A.., 2010. Balance responses to lateral perturbations in human treadmill walking. Journal of Experimental Biology 213, 2655-2664. 
MacKinnon, C.D., Winter, D.A., 1993. Control of whole body balance in the frontal plane during human walking. Journal of Biomechanics 26,633-644.

Townsend, M. A., 1985. Biped gait stabilization via foot placement. Journal of Biomechanics 18,21-38.

Owings, T.M., Grabiner, M.D., 2004. Step width variability, but not step length variability or step time variability, discriminates gait of healthy young and older adults during treadmill locomotion. Journal of Biomechanics 37,935-938.

Murray, M.P., Seireg, A., Scholz, R.C., 1967. Centre of gravity, centre of pressure and supportive forces during human activities. Journal of Applied Physiology 23,831-838.

Prieto, T.E., Myklebust, J.B., Myklebust, B.M., 1993. Characterization and modeling the postural steadiness in the elderly: a review. IEEE Transaction on Rehabilitation Engineering 1, 26-34.

Wagenaar, R.C., Beek, W.J., 2000. Hemiplegic gait: kinematic analysis using walking speed as basis. Journal of Biomechanics 25,1007-1015. 


\section{FIGURE CAPTIONS}

Fig. 1. Experimental apparatus.

Fig. 2. Motions studied: (a) a transient lateral acceleration measured on a train compared with a $0.5 \mathrm{~Hz} 0.5 \mathrm{~ms}^{-2}$ r.m.s. 41/2-cycle motion stimulus employed in study: — measured on a train;-----theoretically generated stimulus. (b) magnitudes and frequencies investigated in the experiment.

Fig. 3. Arrangement of force sensors embedded inside the treadmill.

Fig. 4. Example centre of pressure (COP) and acceleration time histories for a subject walking while exposed to $0.5 \mathrm{~ms}^{-2}$ r.m.s. lateral oscillation at $0.8 \mathrm{~Hz}$ : (a) COP position in the lateral direction; (b) COP velocity in the lateral direction; (c) lateral acceleration measurements: measured acceleration, $=--$ desired acceleration.

Fig. 5. Increase in the reported probability of losing balance with increasing magnitude of oscillation: (a) Effect of acceleration magnitude on the median reported probability of losing balance at each frequency of oscillation (b) Effect of velocity magnitude on the median

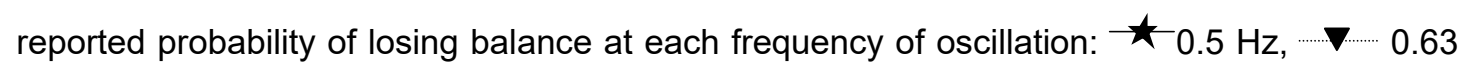
$\mathrm{Hz}, \longrightarrow 0.8 \mathrm{~Hz}, \longrightarrow-1.0 \mathrm{~Hz},-\mathbf{\Delta}-1.25 \mathrm{~Hz}, \longrightarrow-1.6 \mathrm{~Hz}, \multimap 2.0 \mathrm{~Hz}$.

Fig. 6. Effect of frequency on the median reported probability of losing balance (a) at each magnitude of motion acceleration: $\star 0.1, \nabla \ldots 0.125,-0.16, \cdots \cdots \cdots$

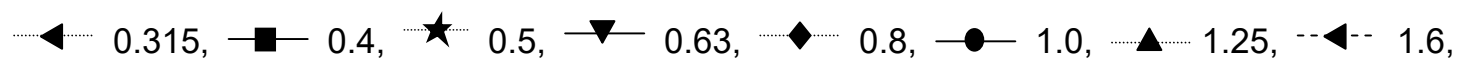

x $2.0 \mathrm{~ms}^{-2}$ r.m.s. (b) at each magnitude of motion velocity: $\rightarrow 0.032, \cdots \nabla \cdots$

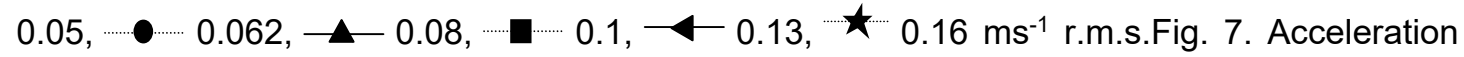
required at each frequency for $25 \%, 50 \%$ and $100 \%$ of 20 people to report their probability of losing balance to be $50 \%$ or greater: $-25 \%$ ( $N=5$ subjects), $\_-50 \%$ ( $N=10$ subjects), $-100 \%(\mathrm{~N}=20$ subjects $)$.

Fig. 8. Effect of acceleration magnitude on the median peak-to-peak lateral COP position at

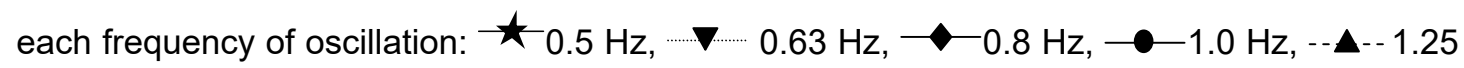
$\mathrm{Hz}, \longrightarrow-1.6 \mathrm{~Hz}, \longleftarrow 2.0 \mathrm{~Hz}$.

Fig. 9. Effect of frequency of oscillation on the median peak-to-peak lateral COP position at each magnitude of motion acceleration: $\star 0.1, \cdots \cdots \cdots, 0.125, \neg 0.16, \cdots \cdots \cdots$

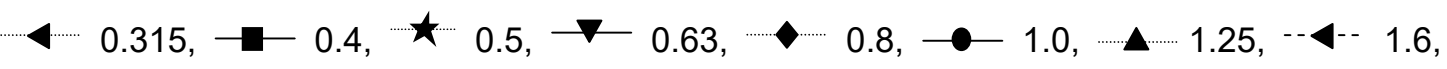
x $2.0 \mathrm{~ms}^{-2}$ r.m.s.

Fig. 10. Effect of frequency of oscillation on the median lateral r.m.s. COP velocity at each

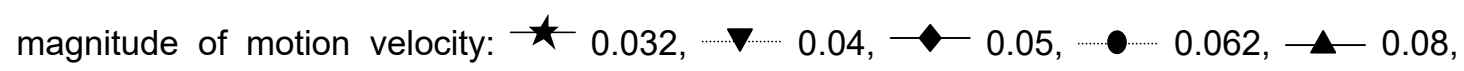
- $0.1, \longleftarrow 0.16 \mathrm{~ms}^{-1}$ r.m.s. 
Fig.11: Effect of velocity of oscillation on the median lateral r.m.s. COP velocity at each frequency of oscillation: $\star 0.5 \mathrm{~Hz}, \boldsymbol{\nabla} 0.63 \mathrm{~Hz}, \longrightarrow 0.8 \mathrm{~Hz}, \longrightarrow-1.0 \mathrm{~Hz},-\mathbf{\Delta}-1.25 \mathrm{~Hz}$, $-1.6 \mathrm{~Hz},-2.0 \mathrm{~Hz}$. 


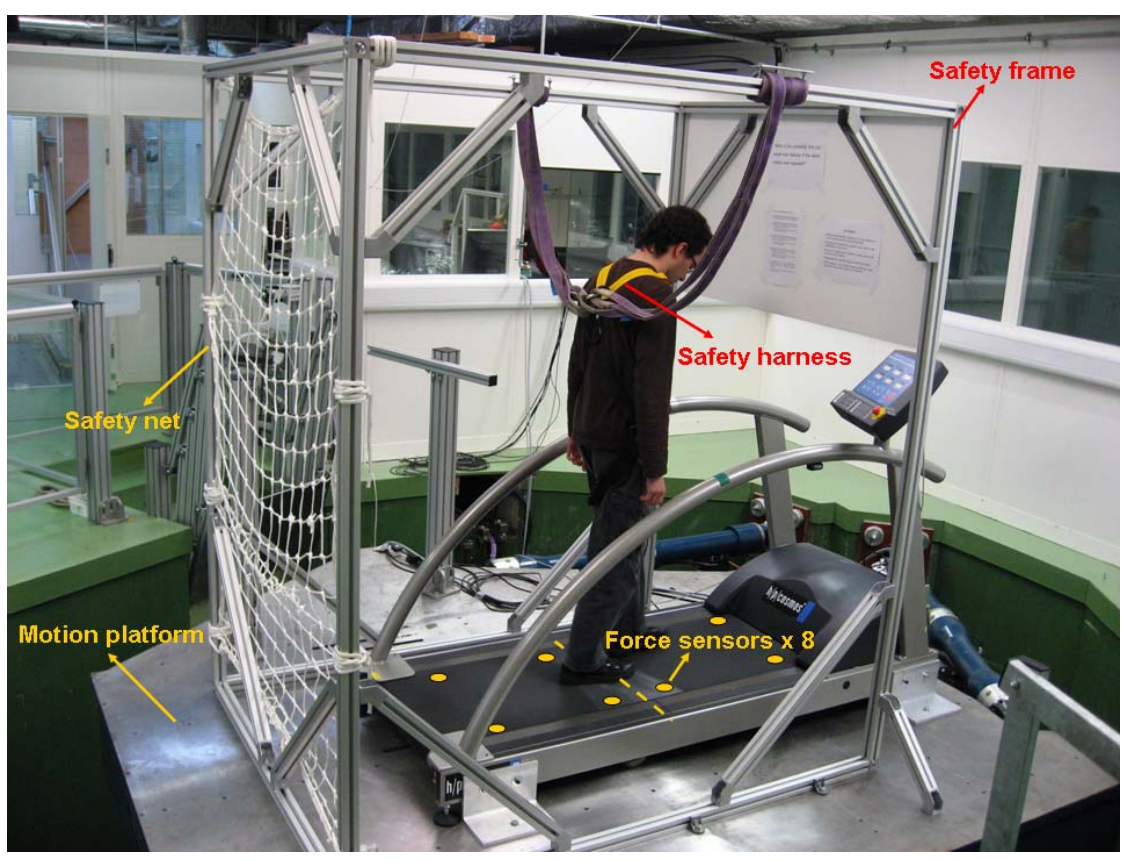

Fig. 1. Experimental apparatus. 

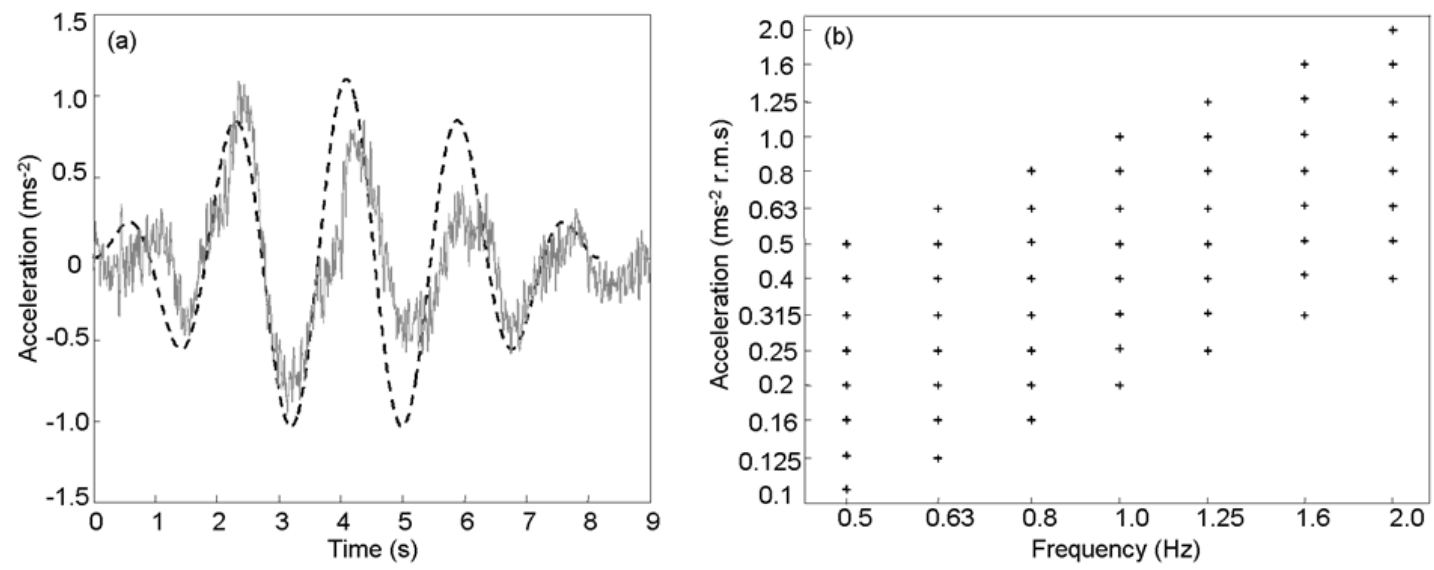

Fig. 2. Motions studied: (a) a transient lateral acceleration measured on a train compared with a $0.5 \mathrm{~Hz} 0.5 \mathrm{~ms}^{-2}$ r.m.s. $41 / 2$-cycle motion stimulus employed in study: measured on a train; ------theoretically generated stimulus. (b) magnitudes and frequencies investigated in the experiment. 


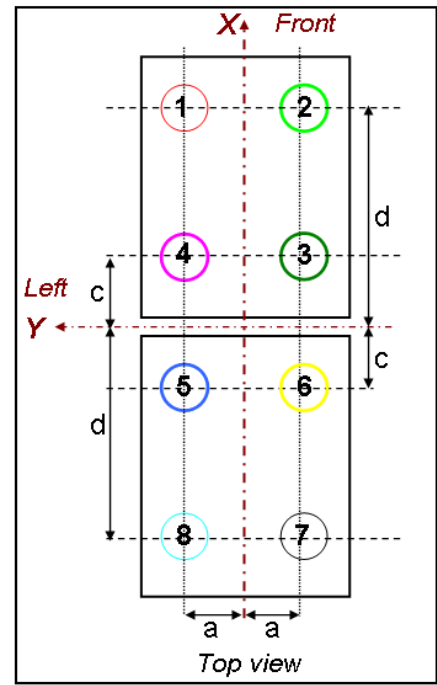

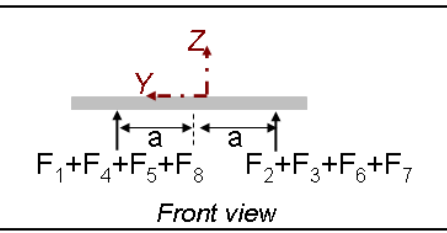

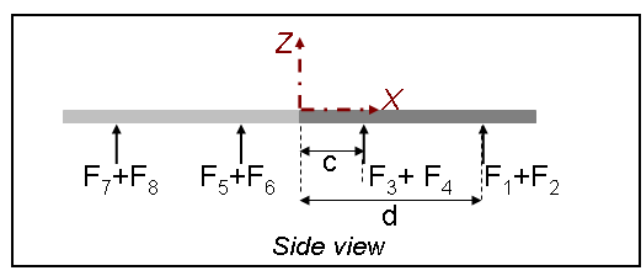

Fig. 3. Arrangement of force sensors embedded inside the treadmill. 

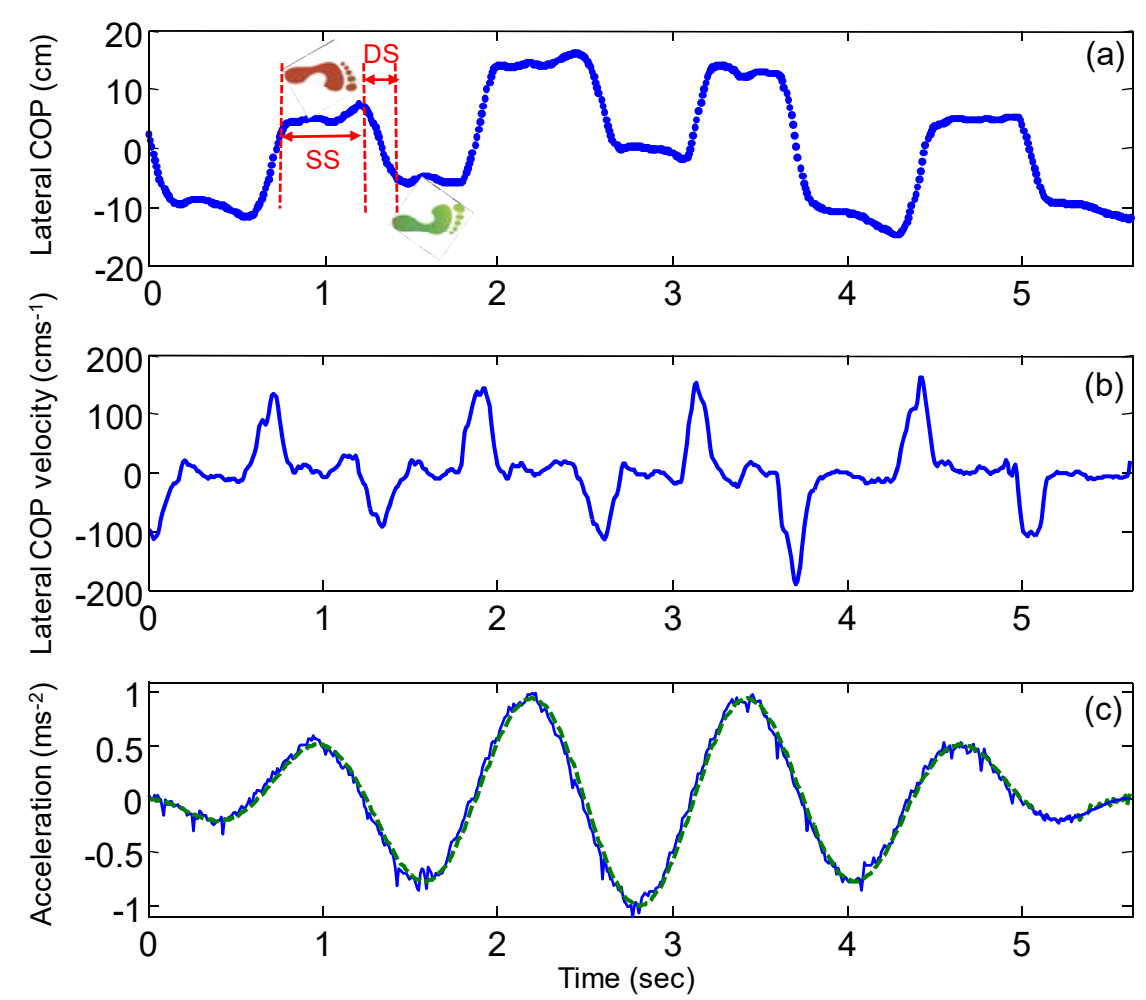

Fig. 4. Example centre of pressure (COP) and acceleration time histories for a subject walking while exposed to $0.5 \mathrm{~ms}^{-2}$ r.m.s. lateral oscillation at $0.8 \mathrm{~Hz}$ : (a) COP position in the lateral direction (SS: single support phase, DS: double support phase of the gait cycle); (b) COP velocity in the lateral direction; (c) lateral acceleration measurements: measured acceleration, $=--$ desired acceleration. 

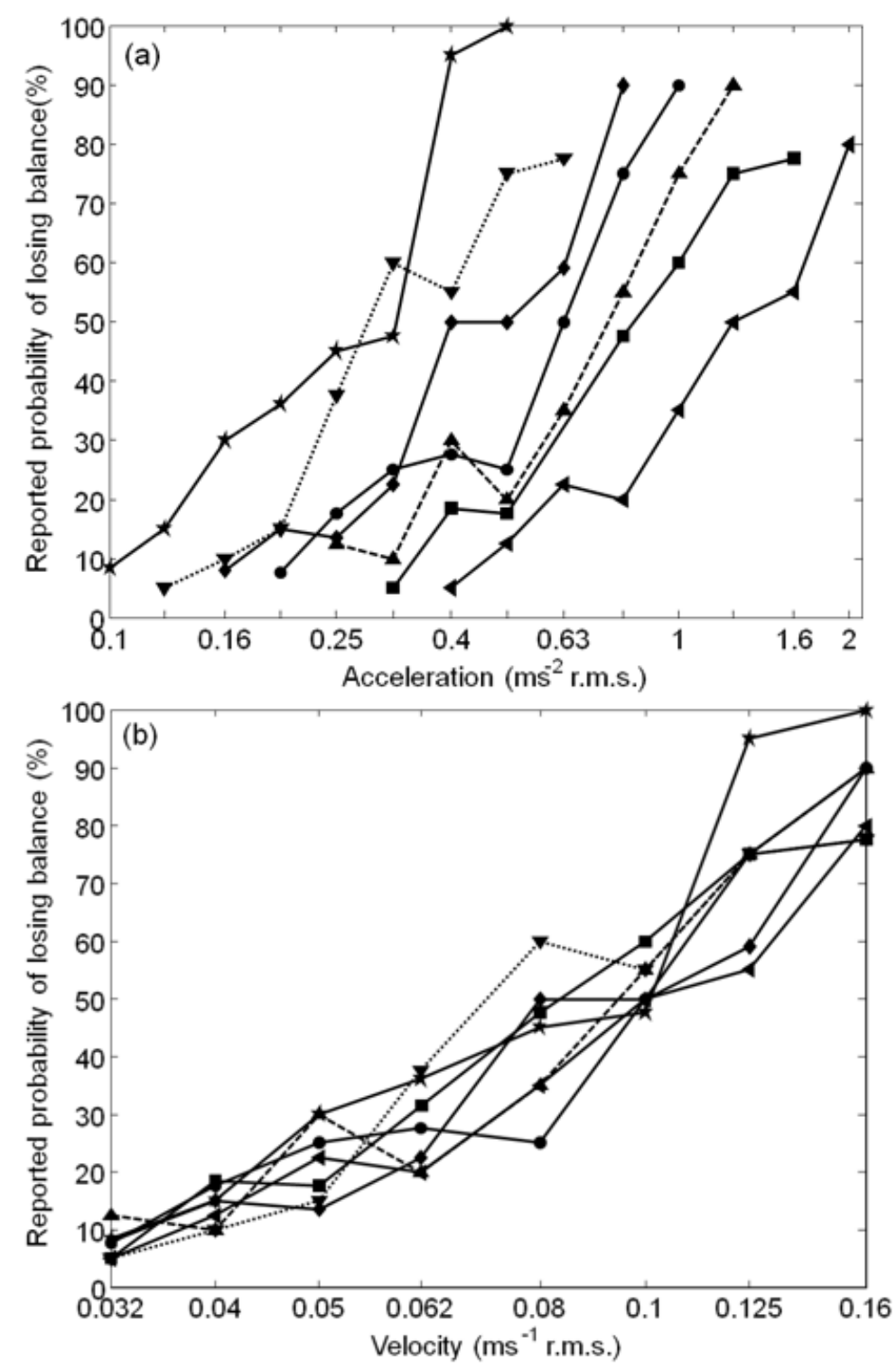

Fig. 5. Increase in the reported probability of losing balance with increasing magnitude of oscillation: (a) Effect of acceleration magnitude on the median reported probability of losing balance at each frequency of oscillation (b) Effect of velocity magnitude on the median reported probability of losing balance at each frequency of oscillation: $\star-0.5 \mathrm{~Hz}, \boldsymbol{\nabla}=0.63$ $\mathrm{Hz}, \longrightarrow 0.8 \mathrm{~Hz}, \longrightarrow-1.0 \mathrm{~Hz},-\mathbf{\Delta}-1.25 \mathrm{~Hz}, \rightarrow-1.6 \mathrm{~Hz}, \longleftarrow 2.0 \mathrm{~Hz}$. 

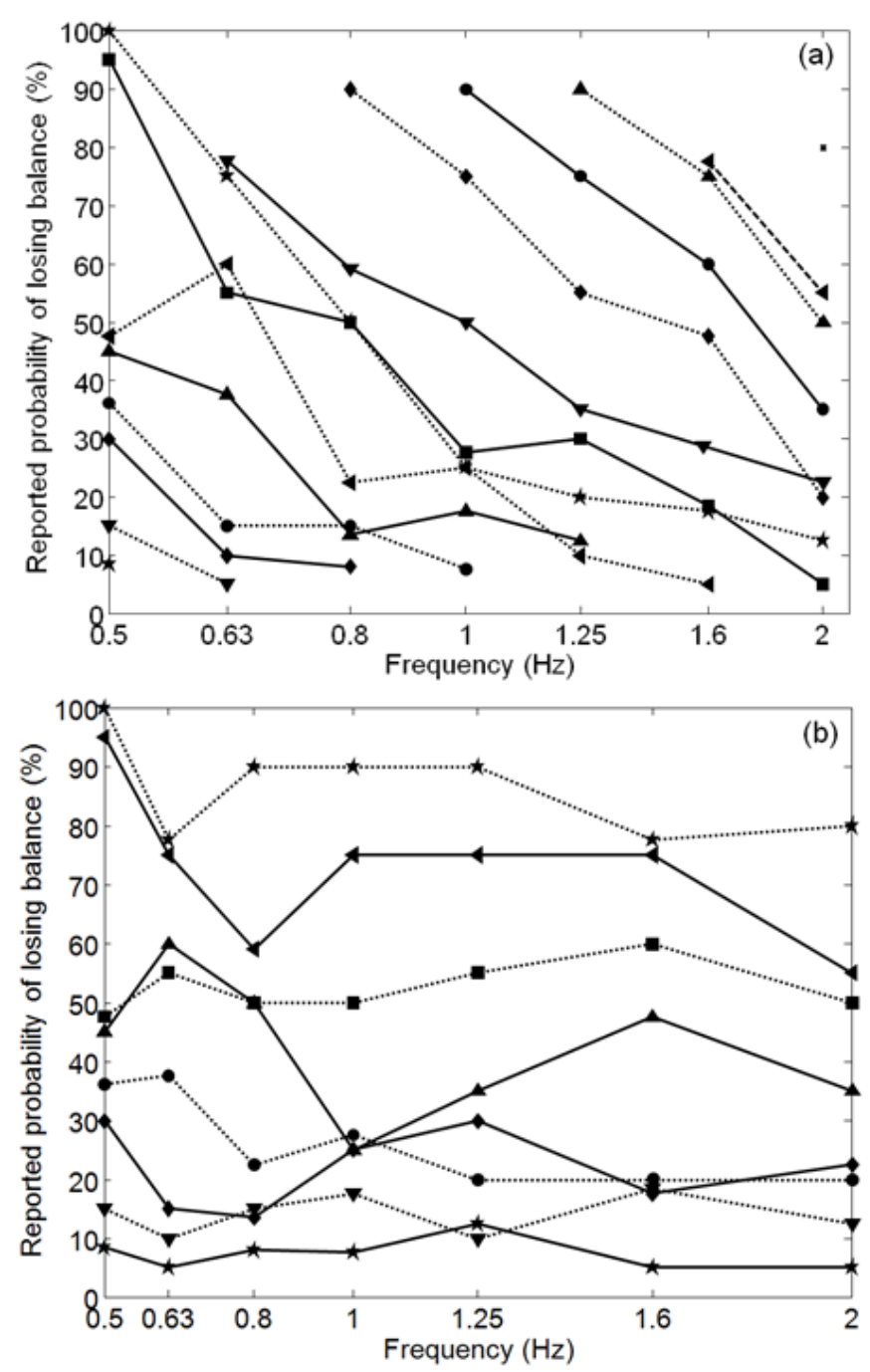

Fig. 6. Effect of frequency on the median reported probability of losing balance (a) at each magnitude of motion acceleration: $\star 0.1, \boldsymbol{\nabla} 0.125, \longrightarrow 0.16, \bullet 0.2,-0.25$, $\ 0.315, \rightarrow-0.4, \star 0.5, \rightarrow 0.63, \rightarrow 0.8, \rightarrow 1.0, \rightarrow 1.25, \cdots-1.6$, x $2.0 \mathrm{~ms}^{-2}$ r.m.s. (b) at each magnitude of motion velocity: $\star-0.032, \nabla 0.04,-$ $0.05, \bullet 0.062, \smile 0.08,-0.1,-0.13, \star 0.16 \mathrm{~ms}^{-1}$ r.m.s. 


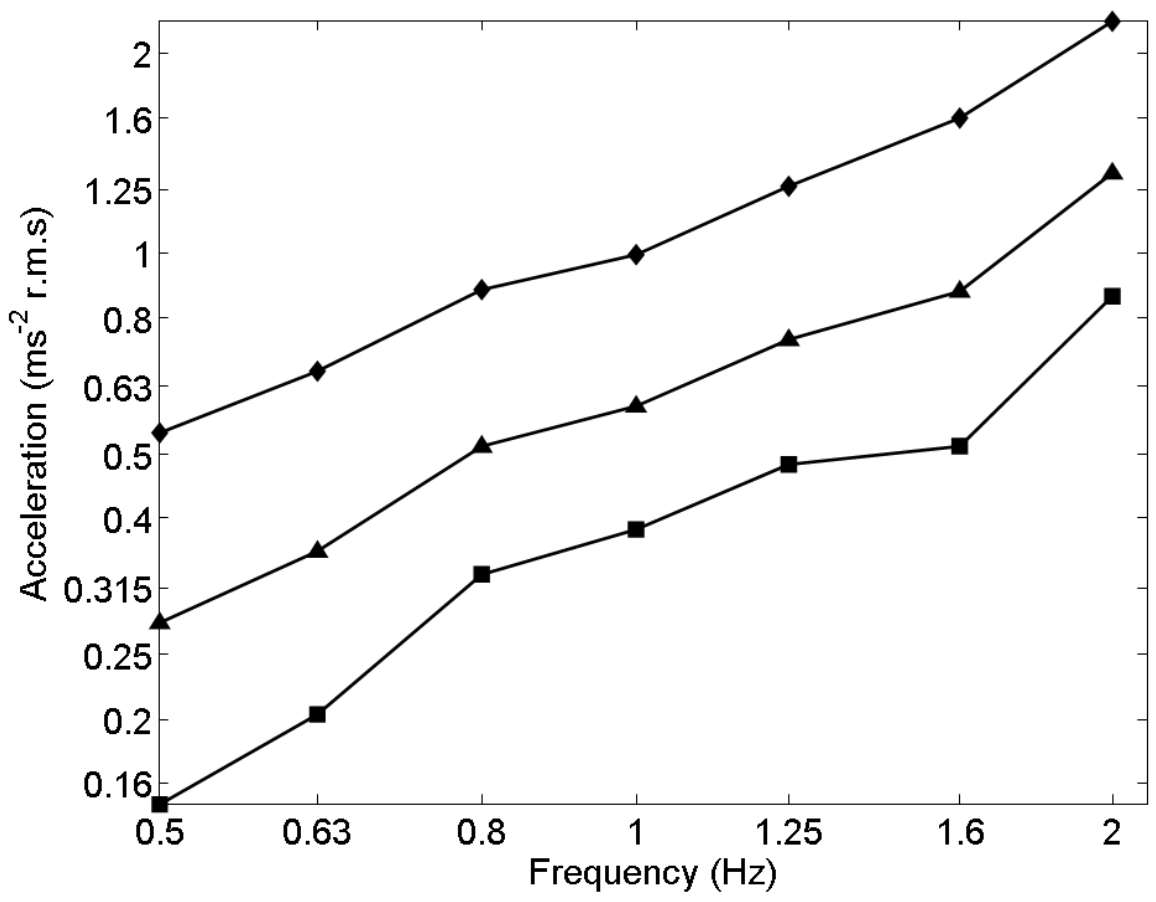

Fig. 7. Acceleration required at each frequency for $25 \%, 50 \%$ and $100 \%$ of 20 people to report their probability of losing balance to be $50 \%$ or greater: $-25 \%$ ( $N=5$ subjects), $\longrightarrow 50 \%$ ( $\mathrm{N}=10$ subjects), $\longrightarrow 100 \%$ ( $\mathrm{N}=20$ subjects). 


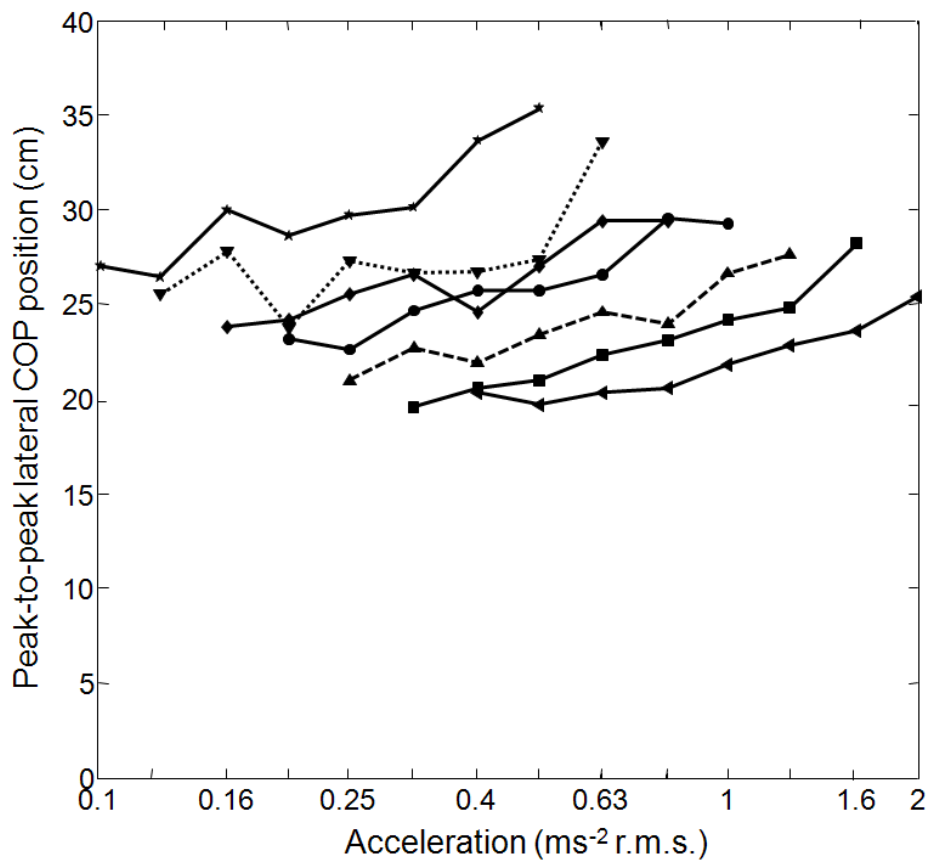

Fig. 8. Effect of acceleration magnitude on the median peak-to-peak lateral COP position at

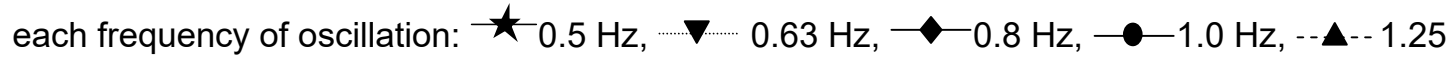
$\mathrm{Hz},-1.6 \mathrm{~Hz}, \longrightarrow 2.0 \mathrm{~Hz}$. 


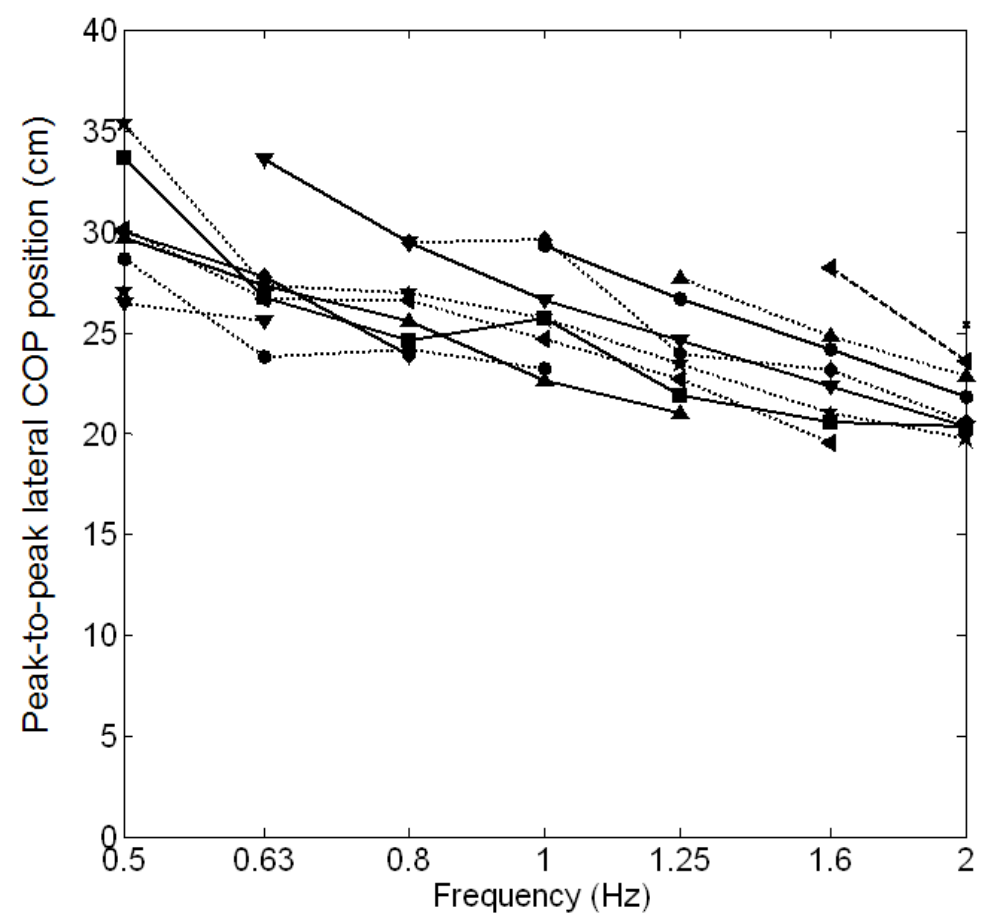

Fig. 9. Effect of frequency of oscillation on the median peak-to-peak lateral COP position at each magnitude of motion acceleration: $\star 0.1, \nabla 0.125, \longrightarrow 0.16, \bullet-0.2, \neg-0.25$, $40.315, \rightarrow 0.4, \star 0.5, \rightarrow 0.63, \rightarrow 0.8, \rightarrow 1.0, \rightarrow 1.25, \cdots<-1.6$, x $2.0 \mathrm{~ms}^{-2}$ r.m.s. 


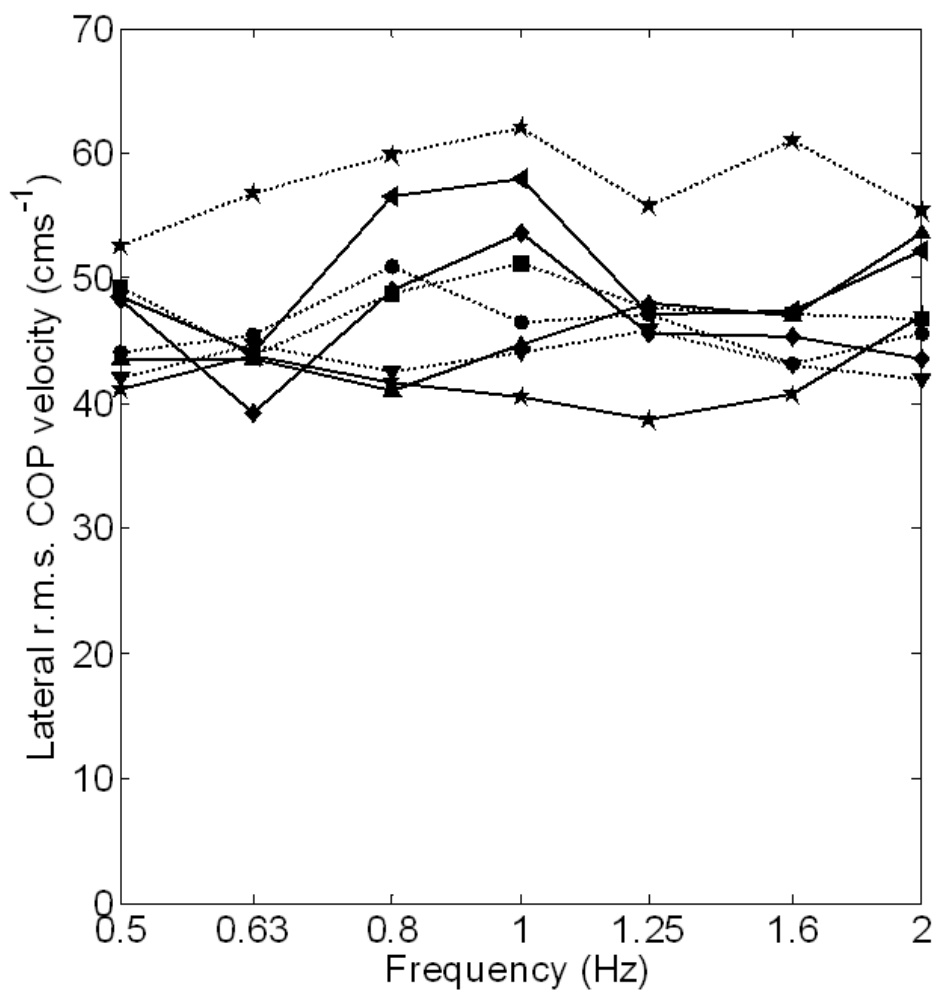

Fig 10. Effect of frequency of oscillation on the median lateral r.m.s. COP velocity at each magnitude of motion velocity: $\star 0.032, \nabla 0.04, \longrightarrow 0.05,-0.062, \neg-0.08$, - $0.1,-0.13, \star 0.16 \mathrm{~ms}^{-1}$ r.m.s. 


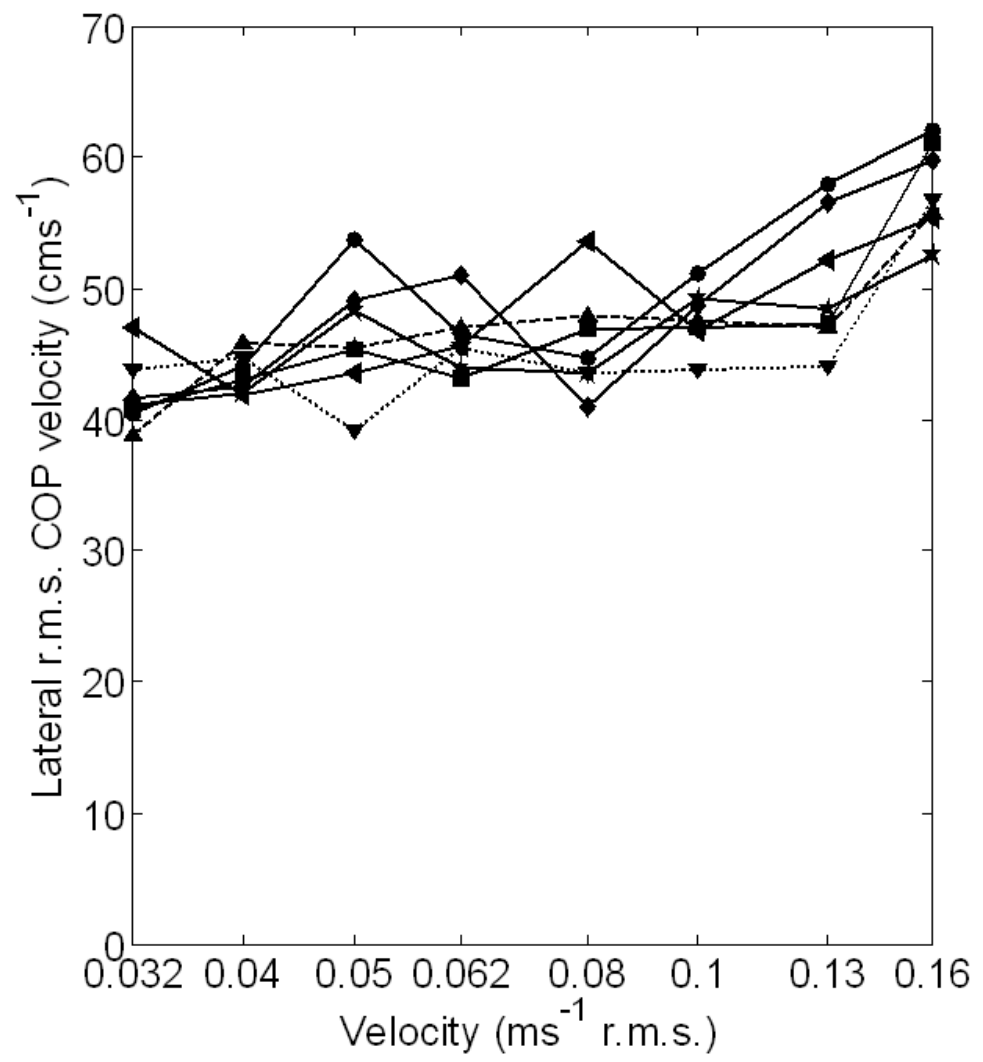

Fig.11. Effect of velocity of oscillation on the median lateral r.m.s. COP velocity at each frequency of oscillation: $\star-0.5 \mathrm{~Hz}, \boldsymbol{\nabla} 0.63 \mathrm{~Hz}, \longrightarrow 0.8 \mathrm{~Hz}, \longrightarrow-1.0 \mathrm{~Hz},--\mathbf{\Delta}-1.25 \mathrm{~Hz}$, $-1.6 \mathrm{~Hz}, \longleftarrow 2.0 \mathrm{~Hz}$. 